\title{
Brillouin Optical Time Domain Analysis Sensor for Active Vibration Control of a Cantilever Beam
}

\author{
Aldo Minardo, Agnese Coscetta, Salvatore Pirozzi, and Luigi Zeni \\ Department of Industrial and Information Engineering, Second University of Naples, 81031 Aversa, Italy \\ Correspondence should be addressed to Agnese Coscetta; agnese.coscetta@unina2.it
}

Received 27 July 2015; Revised 23 October 2015; Accepted 15 November 2015

Academic Editor: Christos Riziotis

Copyright (C) 2016 Aldo Minardo et al. This is an open access article distributed under the Creative Commons Attribution License, which permits unrestricted use, distribution, and reproduction in any medium, provided the original work is properly cited.

The paper reports the use of a distributed optical fiber sensor based on stimulated Brillouin scattering, for structural vibration control. A cantilevered flexible aluminum beam was used as test-bed for vibration control. The proposed approach allows acquiring simultaneously the dynamic strain at several locations. The dynamic strain measured at one (or more) fiber location can be used to implement any vibration control algorithm. Experimental results are reported in which a voice coil, positioned near the fixed end of the cantilever beam, was employed as actuator for the reduction of the vibrations related to the first bending mode of the beam.

\section{Introduction}

A smart structure can be defined as a structure that can sense the external disturbance and response to that with active control in real time to maintain the mission requirements. Smart structures have been designed inspired by living things, in particular by their abilities to adapt their structure, morphology, shape, and properties to the changing environment and aging process. Thus, smart structures have functions that allow them to change their shape, monitor their own health, and in general display adaptive and kinetic features [1-4]. In other words, smart structures not only have traditional structural materials functions, but also have actuating, sensing, and control capabilities. Smart structures are hybrid composite material systems which are composed of three important parts: sensor, actuator, and microprocessor. The sensor senses the variations of the circumstances, and the microprocessor analyzes these signals and then instructs the actuator to change the global mechanical characteristics of the structure. The fiber optic sensor, shape memory alloy, electrorheological fluid, magnetorheological fluid, and piezoelectric materials are sensors and actuators used in smart structure systems [5].

One of the most frequent applications of smart structures is control vibration. Vibration is oscillatory motion of a body or surface about an equilibrium position. It may be characterized in terms of acceleration, velocity, displacement, surface stress or surface strain amplitude, and associated frequency. The frequencies at which vibration naturally occurs and the modal shapes which the vibrating system assumes are properties of the system itself. Any structure can vibrate and will generally do so when excited mechanically or acoustically. Resonance occurs when the frequency of the force input matches the natural frequency of the system, leading to high amplitudes of oscillation at those frequencies. Although high levels of vibration are sometimes useful (e.g., vibrating conveyors and sieves), vibration is generally undesirable, as it often results in excessive noise, mechanical wear, structural fatigue, and possible failure. With currently available analytical tools (e.g., statistical energy analysis, finite element analysis), it is often possible to predict at the design stage the dynamic behavior of a machine and any possible vibration problems. However, vibration problems do appear regularly in new as well as old installations, and vibration control then becomes a remedial exercise instead of the more economic design exercise [6]. Bridges, viaducts, tall buildings, and buildings built near subways and waterways or on reclaimed land often have resonances below $10 \mathrm{~Hz}$ and can oscillate dangerously. The passive-isolation technologies attenuate vibrations within the higher frequency range (above $100 \mathrm{~Hz}$ ), whereas the active-isolation component focused on the lower frequency range $(0$ to $100 \mathrm{~Hz})$ to attenuate the natural low-frequency resonance. Active vibration isolation systems use a combination of sensors and actuators to 
attenuate vibrations. In a feedback active-control system, the forces causing the vibrations (arising from the environment, experimental devices, etc.) are measured by the transducers and the control system sends out-of-phase signals to the actuators in a feedback loop to reduce measured amplitudes. These feedback control systems are reactive, modifying the isolating behavior of the system according to the vibration data measured. There are numerous papers in literature where the active vibration control for flexible structures is tackled (such as [7-10]). Typically, piezoelectric patches, mounted on the structure, are used as actuators. Additional piezoelectric patches or strain-gages are used to sense the vibration level, by measuring the strain in a discrete number of points. Often, also accelerometers are used as vibration sensors [10]. Different identification and control strategies can be applied by exploiting the strain and/or the acceleration measurements. For example, in [7] the pole placement control method is used, while finite element modelling is used in $[8,9]$, by comparing different feedback control strategies. In [10] robust control strategies are presented and tested.

The main limitations of the approaches presented in literature are related to the use of discrete sensors, which allow vibration measurements only in a limited number of points. If additional measurement points are needed to improve the vibration suppression, the cost and complexity of the measuring system progressively increase. Moreover, these points have to be selected at the beginning in order to fix the sensors to the structure, so an optimal placement procedure has to be implemented, which increases the time needed to realize an efficient vibration control.

In general, fiber optic sensors provide a number of advantages over their electrical counterparts, namely, high bandwidth, small size, low weight, corrosion resistance, geometrical flexibility, and inherent immunity to electromagnetic interference. Fiber optic sensors can be embedded in composite materials in a nonobtrusive manner that does not degrade structural integrity. In general, the embedded fiber optic sensors can monitor the health of the structures in service condition. In the last years, several works have demonstrated the possibility of using fiber Bragg grating (FBG) sensors for the active vibration control of smart structures [11-14]. The FBGs are used for active vibration control owing to their small transversal size and the possibility of making an array of many sensors. The small size of the FBGs allows embedding them in composite structures, while the multiplexing capability allows, for example, adopting sensors-averaging techniques [15]. But FBGs are not distributed solution. Moreover, the realization of a single fiber with hundreds of FBGs is a very high-cost solution.

In this paper, we propose the use of distributed optical fiber sensors based on Brillouin scattering as sensor technology to perform active vibration control. Compared to FBGs, distributed optical fiber sensors based on Brillouin scattering do not require any special structure to be fabricated within the fiber (since they use a standard optical fiber) and are intrinsically capable of performing strain measurements in several hundreds or thousands of measuring points simultaneously along the fiber itself. Experiments on a cantilever beam are reported, demonstrating the feasibility of our approach.

\section{Methodology}

Stimulated Brillouin scattering (SBS) in optical fibers allows performing distributed measurements of strain and temperature over large distances and with high spatial resolution. The basic idea underlying the use of SBS for fiber sensors can be understood from the Brillouin frequency shift (BFS) relation:

$$
v_{B}=2 \frac{n V_{a}}{\lambda_{0}},
$$

where $n$ is the effective refractive index of the fiber, $V_{a}$ is the acoustic velocity, and $\lambda_{0}$ is the optical wavelength. As the effective refractive index and the acoustic velocity vary with temperature and strain, the BFS changes in response to local environmental variations. The BFS has a linear dependence on temperature and strain over a wide range, so it can be written as

$$
\Delta \nu_{B}(T, \varepsilon)=C_{\varepsilon} \Delta \varepsilon+C_{T} \Delta T,
$$

where $C_{\varepsilon}$ is the strain coefficient and $C_{T}$ is the temperature coefficient. These coefficients are mostly determined by the fiber composition, pump wavelength, fiber coatings, and jackets. Typical values are $C_{T} \approx 1 \mathrm{MHz} /{ }^{\circ} \mathrm{C}$ and $C_{\varepsilon} \approx$ $500 \mathrm{MHz} / \%$ [16].

In BOTDA sensors, the BFS profile along the fiber is retrieved by recording the interaction between a pulsed pump beam and a counterpropagating stationary probe as a function of time, while scanning the pump-probe spectral shift over a few hundred $\mathrm{MHz}$ range. Any deviation of the local BFS from a reference measurement is a signature of a strain or temperature change. BOTDA sensors allow measuring strain and temperature profiles up to tens of kilometers with a typical strain accuracy of $20 \mu \varepsilon$, a temperature accuracy of $1^{\circ} \mathrm{C}$, and a spatial resolution of $1 \mathrm{~m}$.

The BOTDA setup used for the strain measurements is shown in Figure 1. The light from a distributed feedback laser diode (DFB-LD) operating at $1.55 \mu \mathrm{m}$ wavelength is split into two distinct arms to generate the pump and probe beams. The lower branch in the figure shows the generation of the pulsed pump, while the upper branch shows the generation of the probe signal through double-sideband suppressed-carrier modulation. A polarization scrambler (PS) is employed to average out the Brillouin gain fluctuations associated with variations of state of polarization of the interacting beams. Finally, the fiber Bragg grating (FBG) selects the sideband at lower frequency (Stokes component), while the optical beam reflected by the FBG is detected by a photodetector (PD) and acquired by a data acquisition system (DAQ).

In order to acquire time-varying strain variations at a sufficiently high acquisition rate, the BOTDA setup was operated in the slope-assisted (SA) configuration, that is, at a fixed pump-probe frequency shift $[17,18]$. In brief, by fixing the pump-probe frequency shift at the positive (or negative) slope of the Brillouin gain spectrum (BGS), the 

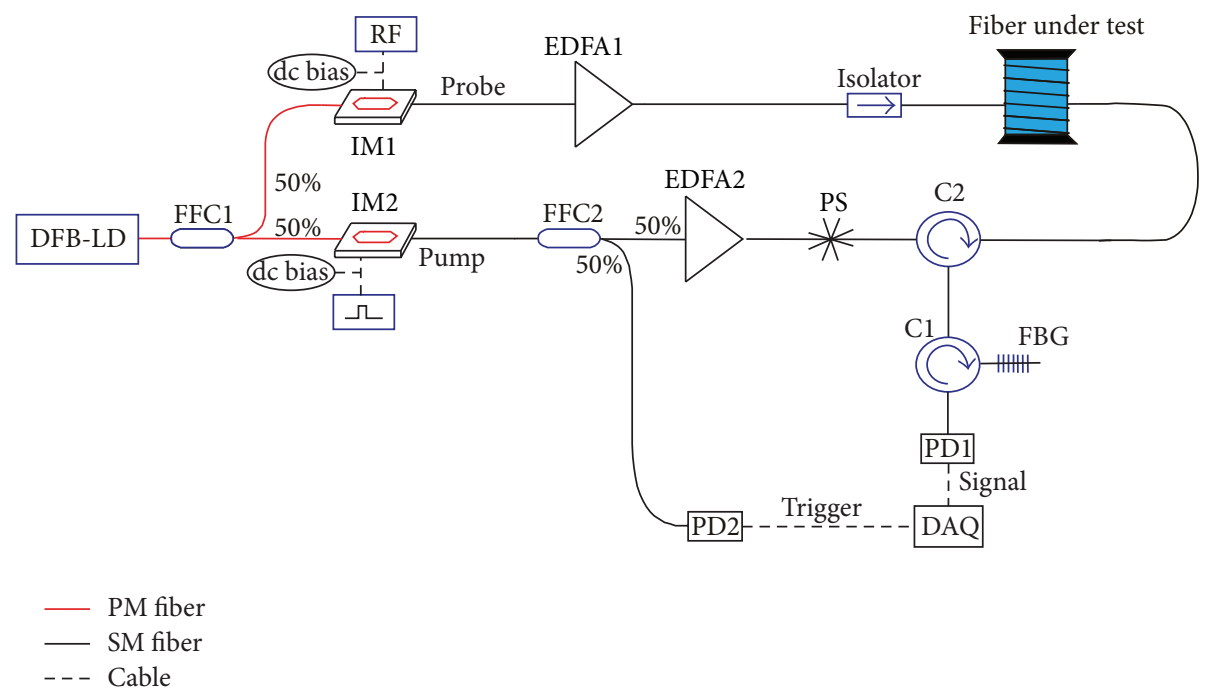

FIGURE 1: Schematic of the BOTDA measurement setup.

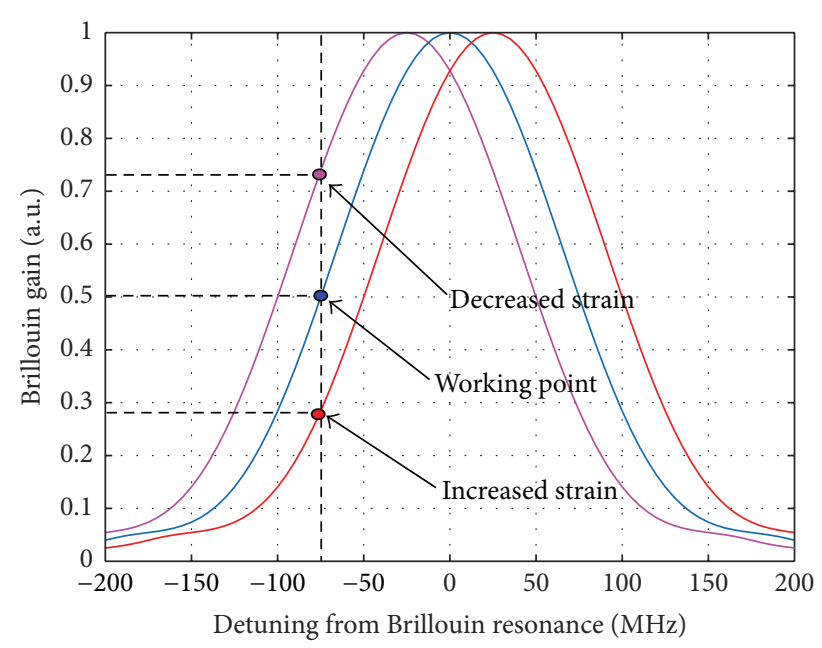

FIgURE 2: Principle of operation of the slope-assisted BOTDA.

probe amplification is modulated by the strain acting on the fiber (see Figure 2). Therefore, by injecting an optical pulse the intensity of the transmitted probe provides an instantaneous measurement of the spatially resolved strain along the fiber, at an acquisition rate only limited by the pulse propagation delay and the number of averages. Note that in the SA-BOTDA method any BFS change due to temperature may displace the operating point from the linear slope of the BGS (see Figure 2), leading to poor operation of the sensor. Therefore, it is necessary to acquire periodically the BGS (by the same setup shown in Figure 1), in order to track the temperature changes and find the optimal pump-probe frequency shift for slope-assisted operation.

\section{Control Scheme and Experimental Results}

The smart structure used as test-bed in this work is constituted by an aluminum cantilever beam, along which a standard single-mode optical fiber was epoxy-glued for dynamic strain measurements. The beam has a length of $1 \mathrm{~m}$, a width of $3 \mathrm{~cm}$, and a thickness of $2 \mathrm{~mm}$. The experimentally determined resonant frequency of the first bending mode is $1.75 \mathrm{~Hz}$.

The BOTDA sensor described in Section 2 was operated with a spatial resolution of $50 \mathrm{~cm}$ (owing to the use of 5-ns pulse duration) and a sampling step of $10 \mathrm{~cm}$ (thus providing up to 10 measuring points along the beam). The number of averages was set to 1024, while repetition rate was set to $100 \mathrm{kHz}$, resulting in a maximum theoretical acquisition rate of about $98 \mathrm{~Hz}$. The actual acquisition rate was $18 \mathrm{~Hz}$, due to the time required by the Digital Signal Processor (DSP) to perform averaging. Anyway, the $18 \mathrm{~Hz}$ rate still guarantees a sufficient bandwidth to correctly measure and control the vibrations associated with the first mode of the beam.

From the vibration control point of view, the optimal positioning of actuators and sensors is fundamental for the proper functioning of the control system and to obtain good performance. A classical approach is based on the use of mathematical models (for simple structures) and/or finite element (FE) models to select the best positions for actuators and sensors, by minimizing and/or maximizing a cost index, related to the controller objective. The use of models can produce results not accurate, especially for complex structures. Moreover, the optimal positions calculated are strongly dependent on the defined cost index. As a consequence, if the cost index changes (e.g., by changing the controller objective) also the positioning of the actuators and sensors changes. In this case, the use of discrete sensors bonded to the structure (such as PZT or strain-gages) does not allow changing the measurement points in order to guarantee the control performance. By using a BOTDA sensor these problems can be overcome. Actually, BOTDA sensors operated in the slopeassisted configuration have been already demonstrated as being capable of performing modal analysis of mechanical structures such as beams or plates $[19,20]$. The use of BOTDA sensor allows analyzing the structure natural modes 


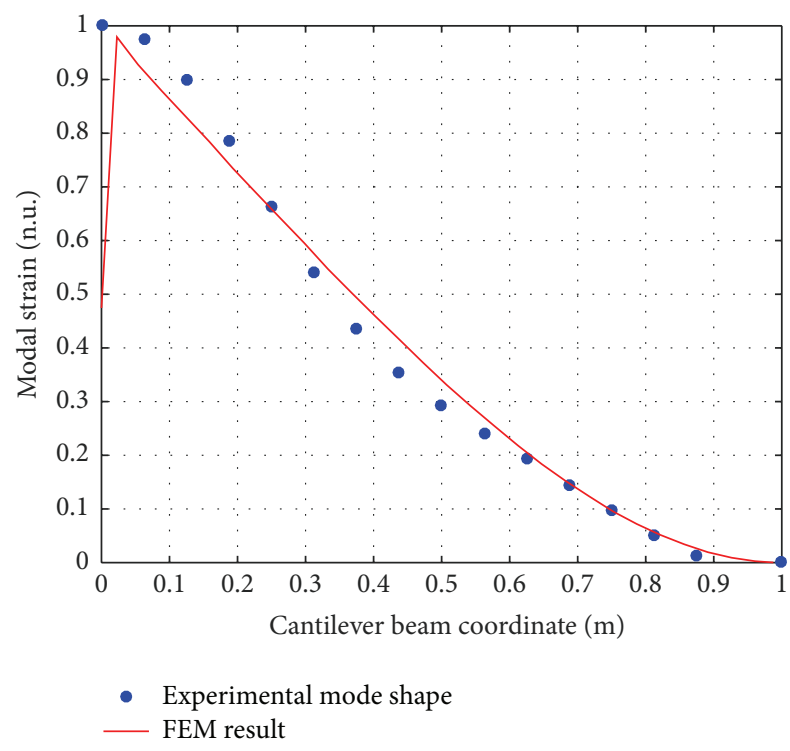

FIgURE 3: Amplitude of strain relative to the first bending mode, as measured by our BOTDA sensor (blue circles), compared to Finite Element Method (FEM) results (red line).

and mode shapes and using the experimental information (instead of mathematical or FE models) to select the actuator positioning (see Figure 3).

After this phase, the same sensor can be used to implement the vibration control law without the need to solve the problem of the optimal positioning of the sensors, thanks to its intrinsic distributed nature. In particular, in BOTDA sensors the user can change the measurement points to use in the feedback loop without any additional effort. Also, combination of different measurement points can be used. Hence, the user can select how many and which measurement point should be used in the feedback loop on the basis of the controller objective and if the objective changes the user can select new points in order to guarantee always the maximum control performance.

The objective of this paper is to show that the BOTDA sensor can be effectively used for vibration control. It has been experimentally tested for the reduction of first vibrational mode of the test-bed. The control scheme is reported in Figure 4, where the smart structure has been already described. The strain measured along the beam (10 measurement points at $10 \mathrm{~cm}$ step) by the BOTDA sensor is available for control purposes. The disturbance acts on the beam tip. All modes are excited but the control objective is to reduce the first mode amplitude. Since only one mode has to be controlled, a single actuation point is sufficient. A voice coil has been selected to apply the punctual force necessary to reduce the vibration. In this case the actuator was positioned close to the fixed end (Figure 5), since it is the optimal point for the control of the first bending mode of the beam, as well known from the literature. The voice coil used in the tests, manufactured by Moticont (code GVCM-051051-01), was characterized by a maximum continuous force of $28 \mathrm{~N}$ and a force constant of $8 \mathrm{~N} / \mathrm{A}$. A transconductance

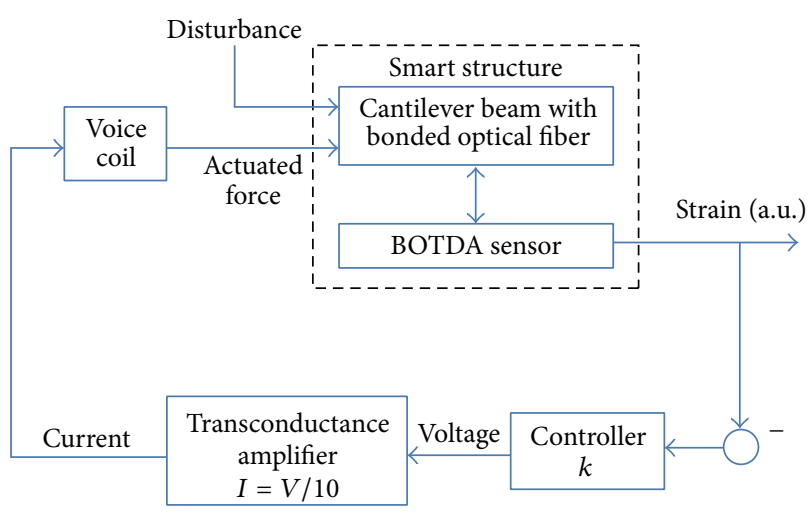

FIgURE 4: Block diagram of the control loop.

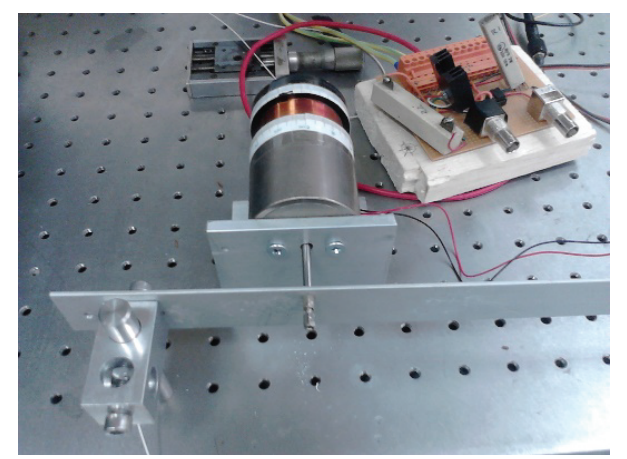

FIGURE 5: Voice coil bolted to the cantilever beam.

amplifier has been used to compute the voice coil current from the voltage control signal computed by the controller. Both data acquisition and computation of the control signal were performed via custom Matlab code running under Windows Operating System. The control signal has been interfaced with the amplifier by using a 16-bit D/A converter.

In this work, a standard controller $k$ proportional to the measured strain coming from a point along the structure has been implemented. By indicating with $u(t)$ the control input (amplifier input) and with $s_{i}(t)$ the strain measurement coming from the $i$ th measuring point from the BOTDA sensor the following control signal can be selected:

$$
u(t)=-k s_{i}(t), \quad i \in 1, \ldots, 10,
$$

where the selection of $i$ corresponds to the section of the measurement point used for the feedback (i.e., the position of the sensor).

In the proposed test-bed, from the stability point of view, only one of the measurement points is colocated with the actuator position, while the other nine points are not colocated. It is well known from literature $[21,22]$ that the use of colocated actuator/sensor pairs guarantees a minimum phase system, which allow increasing the control gain and obtaining better performance with good stability margins. Instead, the use of noncolocated sensing points limits the control action. For the experiments described below, the measurement point $i=2$ has been selected for the feedback, being the point colocated with the actuator. 


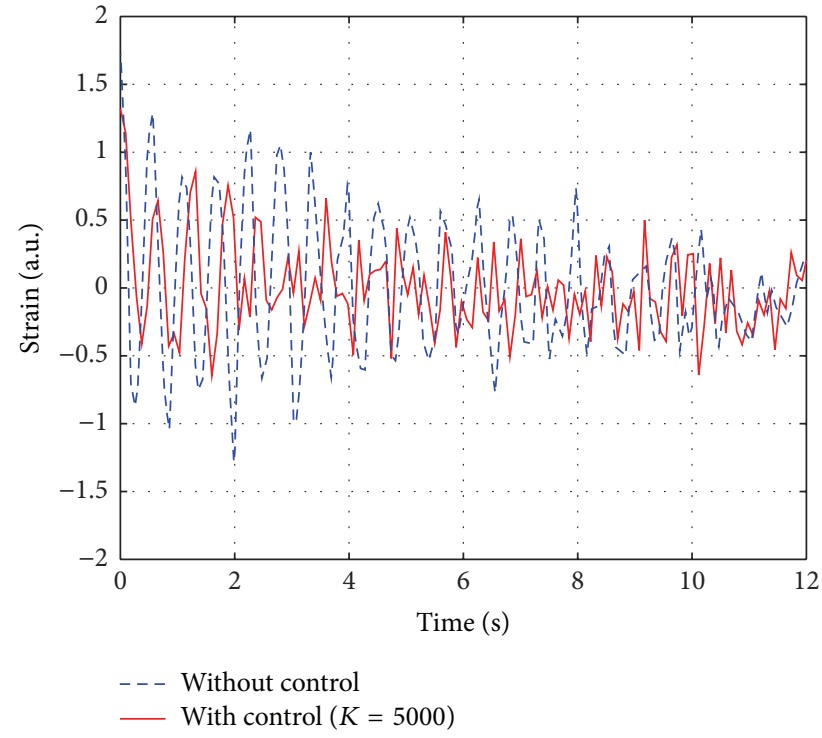

(a)

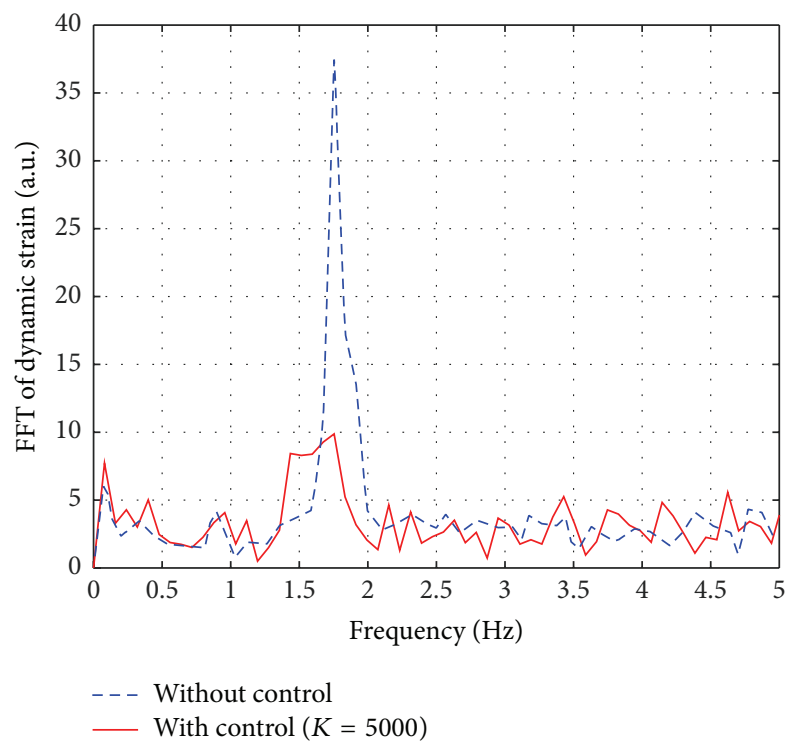

(b)

Figure 6: Comparison of time (a) and frequency (b) free response in both controlled and no control cases.

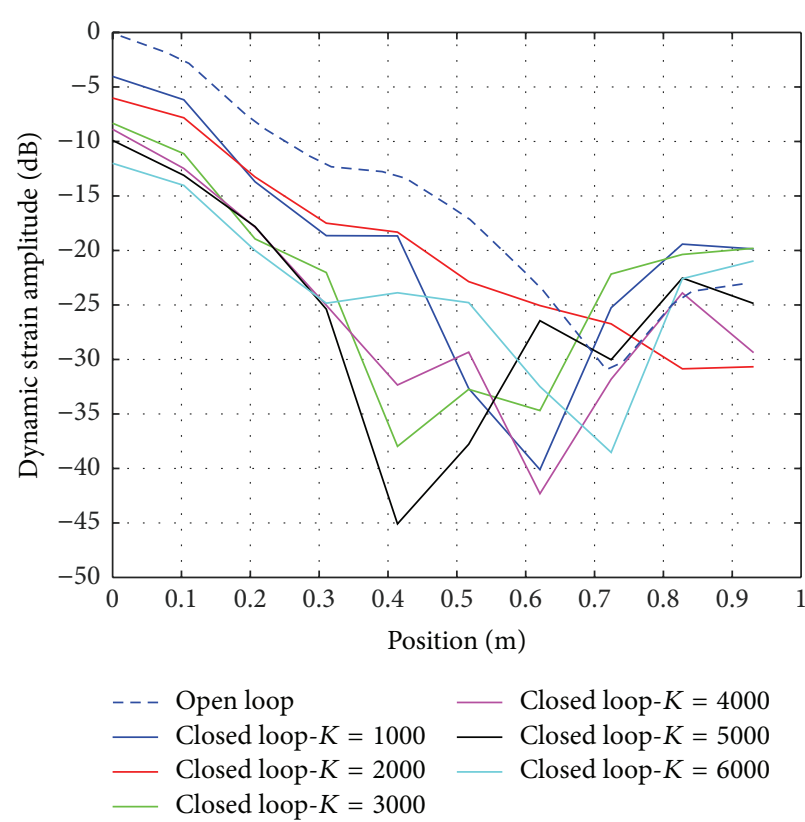

(a)

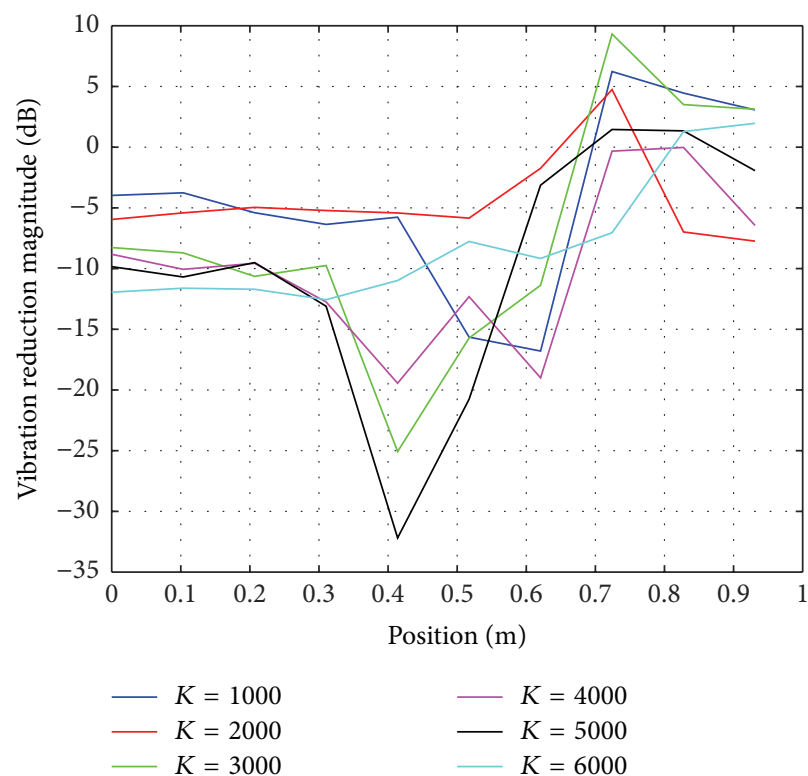

(b)

Figure 7: Dynamic strain (a) and vibration reduction (b) magnitude, as a function of position along the beam and for various controller gains.

To show the effectiveness of the proposed approach, different open loop and closed loop cases are compared. The free response of the beam in the open loop and closed loop cases has been compared, starting from the same initial condition (free end deflection equal to $5 \mathrm{~cm}$ ), in the time domain and in the frequency domain. In these tests, a value of $k=5000$ as proportional control algorithm was used. The controller optimal gain was experimentally evaluated, by measuring the vibration reduction magnitude of the first vibrational mode all along the cantilever at varying gain values.

In Figure 6(a) we show the strain free response measured by the sensor, in the time domain, for the point used as controller input, without and with the control activated. The corresponding Fourier spectra are shown in Figure 6(b). It is seen that a reduction of about $75 \%$ of the strain amplitude at the resonance frequency of the first mode $(1.75 \mathrm{~Hz})$ is achieved with control. 
In order to evaluate the effectiveness of our control scheme, we have repeated the measurement at various control gains and evaluated the performance along the whole structure. Figure 7(a) shows the strain amplitude as measured by the sensor at each position in the open loop (control off) and in the closed loop cases, for different controller gains. We also report, in Figure 7(b), the magnitude of vibration reduction along the beam. It is seen that a maximum reduction of vibration (in terms of strain) as large as $\sim 32 \mathrm{~dB}$ was achieved, for a controller gain equal to 5000 and a beam position close to the middle. We observe from Figure $7(\mathrm{~b})$ that the ability to damp the vibration decreases in the last $\sim 30 \mathrm{~cm}$ of the beam. This depends on the strain associated with the mode shape of the controlled mode, which tends to vanish in proximity of the free end; thus in that region no control action is needed.

\section{Conclusion and Future Work}

Vibration reduction of the first mode of a vibrating cantilever beam was demonstrated by using a distributed optical fiber sensor based on stimulated Brillouin scattering and a voice coil actuator. The proposed approach uses a single optical fiber as the sensing element, allowing obtaining the dynamic strain along the entire length of the beam simultaneously. Future work will be focused on the possibility of improving the quality of feedback signals exploiting the large number of available measuring points. In particular, sensor-averaging techniques may be used to filter out undesired resonance modes without adversely affecting phase and amplitude. Moreover, the conditioning electronics used to implement the BOTDA sensor can be improved in order to increase also the spatial resolution of the strain measurements. More complex control algorithm will be tested in order to demonstrate the effectiveness of the BOTDA sensor for vibration control of multiple modes and complex structures.

\section{Conflict of Interests}

The authors declare that there is no conflict of interests regarding the publication of this paper.

\section{References}

[1] M. V. Gandhi and B. S. Thompson, Smart Materials and Structures, Chapman and Hall, London, UK, 1992.

[2] E. Udd, Fiber Optic Smart Structures, John Wiley \& Sons, New York, NY, USA, 1995.

[3] S. Y. Du and J. S. Leng, "A study on the optical fibre intelligent composite materials and its monitoring system," Advances in Mechanics, vol. 4, pp. 496-503, 1992.

[4] S. Y. Du and J. S. Leng, "Fiber optical NDE method for advanced composites," Aerospace Materials \& Technology, vol. 5, pp. 14-18, 1992.

[5] A. Selvarajan and A. Asoundi, "Photonics, fiber optic sensors and their applications in smart structures," Journal of Nondestructive Evaluation, vol. 15, no. 2, pp. 41-56, 1995.

[6] D. A. Bias and C. H. Hansen, Engineering Noise Control: Theory and Practice, CRC Press, 4th edition, 2009.
[7] W. J. Manning, A. R. Plummer, and M. C. Levesley, "Vibration control of a flexible beam with integrated actuators and sensors," Smart Materials and Structures, vol. 9, no. 6, pp. 932-939, 2000.

[8] P. Gaudenzi, R. Carbonaro, and E. Benzi, "Control of beam vibrations by means of piezoelectric devices: theory and experiments," Composite Structures, vol. 50, no. 4, pp. 373-379, 2000.

[9] I. Bruant, G. Coffignal, F. Léné, and M. Vergé, "Active control of beam structures with piezoelectric actuators and sensors: modeling and simulation," Smart Materials and Structures, vol. 10, no. 2, pp. 404-408, 2001.

[10] A. Cavallo, G. De Maria, C. Natale, and S. Pirozzi, "Robust control of flexible structures with stable bandpass controllers," Automatica, vol. 44, no. 5, pp. 1251-1260, 2008.

[11] K. K. Chau, B. Moslehi, G. Song, and V. Sethi, "Experimental demonstration of fiber Bragg grating strain sensors for structural vibration control," in Smart Structures and Materials 2004: Sensors and Smart Structures Technologies for Civil, Mechanical, and Aerospace Systems, vol. 5391 of Proceedings of SPIE, p. 753, San Diego, Calif, USA, July 2004.

[12] C. Ambrosino, G. Diodati, A. Laudati et al., "Active vibration control using fiber Bragg grating sensors and piezoelectric actuators in co-located configuration," in Proceedings of the 3rd European Workshop on Optical Fibre Sensors, vol. 6619 of Proceedings of SPIE, Napoli, Italy, July 2007.

[13] G. Cazzulani, S. Cinquemani, and L. Comolli, "Enhancing active vibration control performances in a smart structure by using fiber Bragg gratings sensors," in Sensors and Smart Structures Technologies for Civil, Mechanical, and Aerospace Systems, vol. 8345 of Proceedings of SPIE, San Diego, Calif, USA, March 2012.

[14] S. Cinquemani, G. Cazzulani, and F. Braghin, "Vibration control of shell-like structures with optical strain sensors," in Active and Passive Smart Structures and Integrated Systems, vol. 9057 of Proceedings of SPIE, San Diego, Calif, USA, March 2014.

[15] G. Cazzulani, S. Cinquemani, L. Comolli, and F. Resta, "A quasimodal approach to overcome FBG limitations in vibration control of smart structures," Smart Materials and Structures, vol. 22, no. 12, Article ID 125002, 2013.

[16] M. Niklès, L. Thévenaz, and P. A. Robert, "Brillouin gain spectrum characterization in single-mode optical fibers," Journal of Lightwave Technology, vol. 15, no. 10, pp. 1842-1851, 1997.

[17] R. Bernini, A. Minardo, and L. Zeni, "Dynamic strain measurement in optical fibers by stimulated brillouin scattering," Optics Letters, vol. 34, no. 17, pp. 2613-2615, 2009.

[18] Y. Peled, A. Motil, I. Kressel, and M. Tur, "Fast and distributed brillouin sensing for dynamic SHM," in Proceedings of the 6th European Workshop on Structural Health MonitoringWe.2.C.3, 2012.

[19] A. Minardo, A. Coscetta, S. Pirozzi, R. Bernini, and L. Zeni, "Modal analysis of a cantilever beam by use of Brillouin based distributed dynamic strain measurements," Smart Materials and Structures, vol. 21, no. 12, Article ID 125022, 2012.

[20] A. Minardo, A. Coscetta, R. Bernini et al., "Structural damage identification in an aluminum composite plate by brillouin sensing," IEEE Sensors Journal, vol. 15, no. 2, pp. 659-660, 2015.

[21] A. Cavallo, G. De Maria, C. Natale, and S. Pirozzi, Active Control of Flexible Structures-From Modelling to Implementation, Springer, London, UK, 2010.

[22] J. M. Maciejowski, Multivariable Feedback Design, AddisonWesley, 1989. 


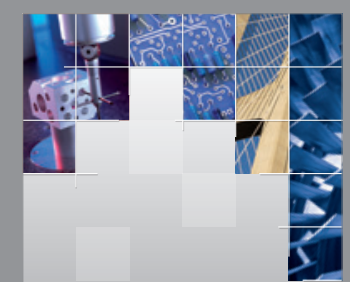

\section{Enfincering}
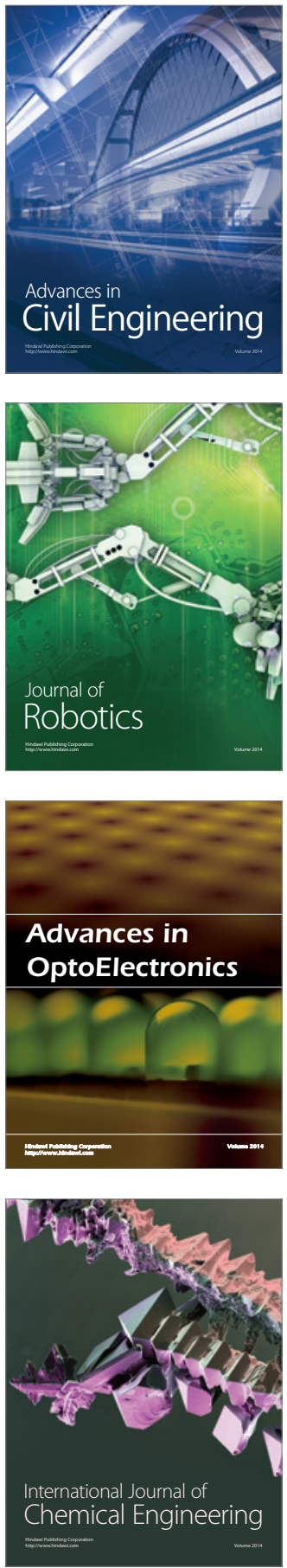

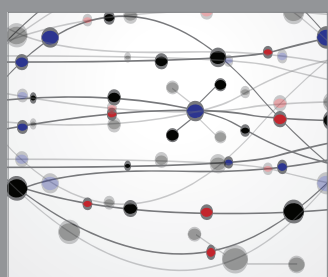

The Scientific World Journal

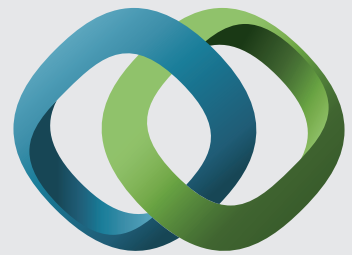

\section{Hindawi}

Submit your manuscripts at

http://www.hindawi.com
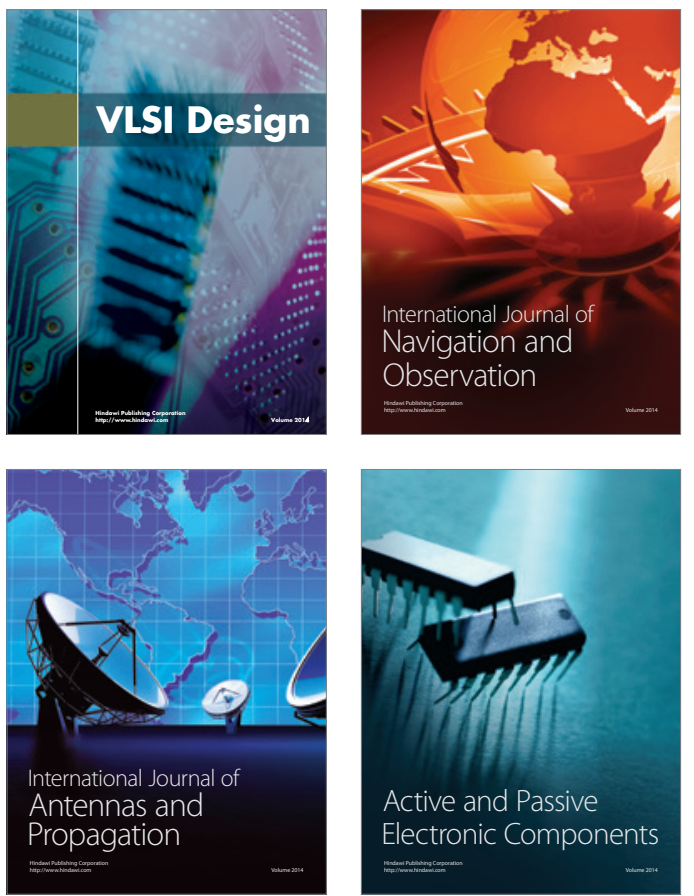
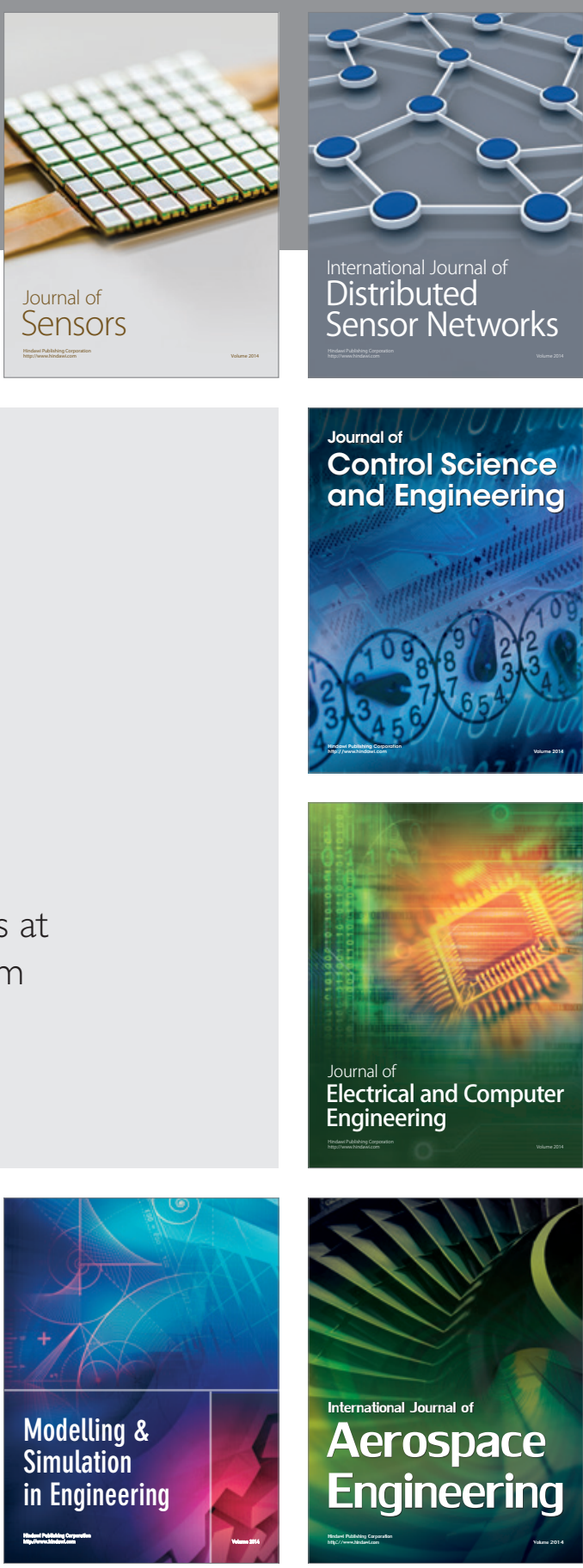

International Journal of

Distributed

Sensor Networks

Journal of

Control Science

and Engineering
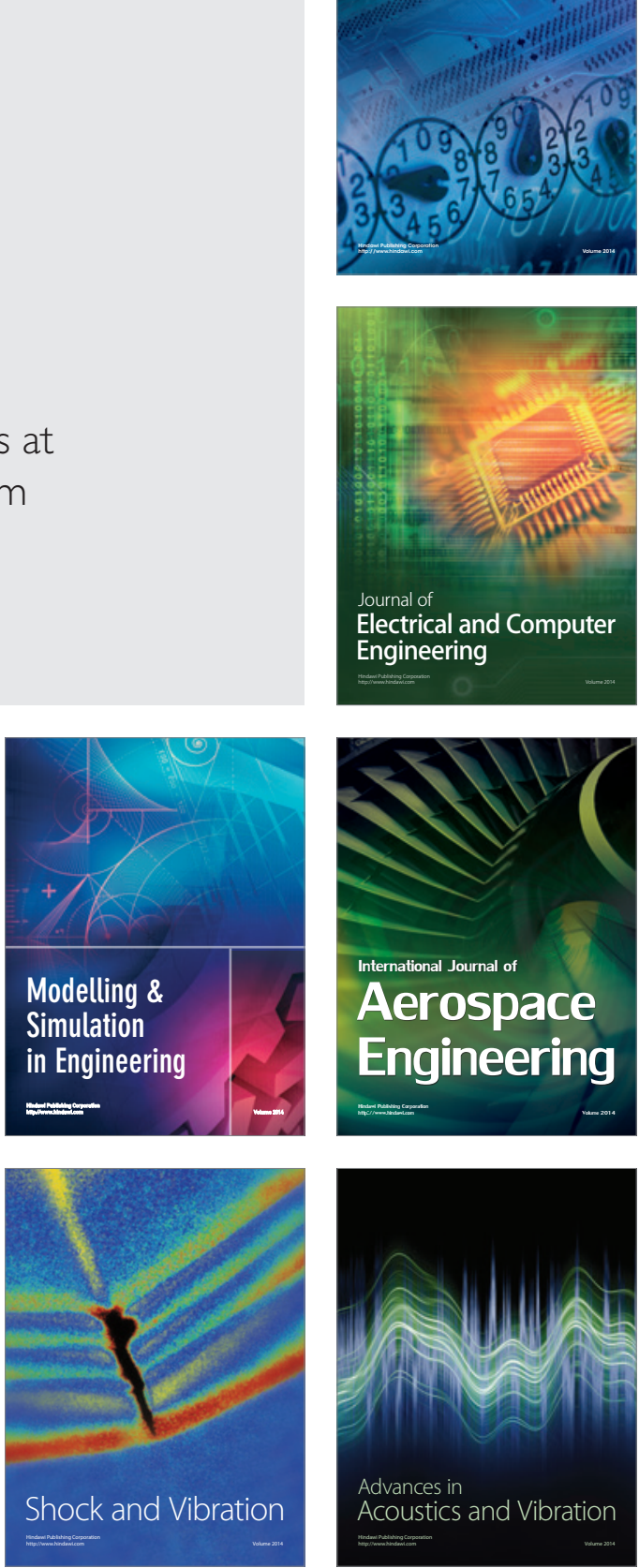